\title{
On the Existence of Bright Solitons in Cubic-Quintic Nonlinear Schrödinger Equation with Inhomogeneous Nonlinearity
}

\author{
Juan Belmonte-Beitia ${ }^{1,2}$ \\ ${ }^{1}$ Departamento de Matemáticas, E.T.S de Ingenieros Industriales, Universidad de Castilla-La Mancha, \\ 13071 Ciudad Real, Spain \\ ${ }^{2}$ Instituto de Matemática Aplicada a la Ciencia y la Ingeniería (IMACI), Universidad de Castilla-La \\ Mancha, 13071 Ciudad Real, Spain
}

Correspondence should be addressed to Juan Belmonte-Beitia, juan.belmonte@uclm.es

Received 21 April 2008; Revised 3 July 2008; Accepted 4 July 2008

Recommended by Mehrdad Massoudi

We give a proof of the existence of stationary bright soliton solutions of the cubic-quintic nonlinear Schrödinger equation with inhomogeneous nonlinearity. By using bifurcation theory, we prove that the norm of the positive solution goes to zero as the parameter $\lambda$, called chemical potential in the Bose-Einstein condensates' literature, tends to zero. Moreover, we solve the time-dependent cubicquintic nonlinear Schrödinger equation with inhomogeneous nonlinearities by using a numerical method.

Copyright (C) 2008 Juan Belmonte-Beitia. This is an open access article distributed under the Creative Commons Attribution License, which permits unrestricted use, distribution, and reproduction in any medium, provided the original work is properly cited.

\section{Introduction}

Nonlinear Schrödinger (NLS) equations appear in a great array of contexts [1], for example, in semiconductor electronics [2,3], optics in nonlinear media [4], photonics [5], plasmas [6], the fundamentation of quantum mechanics [7], the dynamics of accelerators [8], the mean field theory of Bose-Einstein condensates [9, 10], or in biomolecule dynamics [11]. In some of these fields and in many others, the NLS equation appears as an asymptotic limit for a slowly varying dispersive wave envelope propagating a nonlinear medium [12].

The study of these equations has served as the catalyzer for the development of new ideas or even mathematical concepts such as solitons [13] or singularities in partial differential equations [14, 15].

In the last years, there has been an increased interest in a variant of the standard nonlinear Schrödinger equation, that is, the so-called nonlinear Schrödinger equation with 
inhomogeneous nonlinearity, which is

$$
i \psi_{t}=-\psi_{x x}+g(x)|\psi|^{2} \psi,
$$

with $x \in \mathbb{R}$, where $\psi(t, x)$ is a complex valued function and $g(x)$ is a real function.

This equation arises in different physical contexts such as nonlinear optics and dynamics of Bose-Einstein condensates with Feschbach resonance management [16-26]. Different aspects of the dynamics of solitons in these contexts have been studied such as the emission of solitons $[16,17]$ and the propagation of solitons when the space modulation of the nonlinearity is a random [18], periodic [22], linear [19], or localized function [21]. Equation (1.1) admits special solutions called standing waves, solitary waves, or bright solitons of the form $\psi(t, x)=u(x) e^{i \lambda t}$, where the profile $u$ is time-independent. The function $u$ satisfies

$$
-u_{x x}+\lambda u+g(x) u^{3}=0 .
$$

The study of the existence of decaying solutions for equations or systems like (1.2) has gained the interest of many mathematicians in recent years. Many results are available for semilinear elliptic equations in $\mathbb{R}^{N}$. Without being exhaustive, we refer to [27-35].

At this point, it should be noticed that a way to get compactness in semilinear elliptic problems in unbounded domains is to assume the invariance of the coefficients under a compact group of symmetries. Indeed, dealing with the following equation:

$$
-\Delta u+a(x) u=b(x)|u|^{p-1} u
$$

with $x \in \mathbb{R}^{N}$, Strauss radial compact imbedding (see, e.g., [36]) implies the existence of a positive radial ground state as soon as $a$ and $b$ are radially symmetric, positive, and bounded. More sophisticated conditions have been exploited, for example, in [37]. However, these results do not apply to the one-dimensional case. Indeed, assuming radial symmetry means that the coefficients $a$ and $b$ are even functions. One can therefore look for even solutions, but $H^{1}(0,+\infty)$ do not have better compactness properties than $H^{1}(\mathbb{R})$. Nevertheless, symmetry is always a simplifying condition and it has been extensively used for finding connecting orbits in reversible Hamiltonian systems [38]. In [39], a unique positive homoclinic solution is obtained for the model equation

$$
-u^{\prime \prime}+a(x) u=b(x) u^{3},
$$

assuming that $a$ and $b$ are even and bounded from below by a positive constant such that $x a^{\prime}(x)>0$ and $x b^{\prime}(x)<0$, for every $x \neq 0$. Analytical solutions of (1.4) have been calculated in $[40,41]$ for different functions $a$ and $b$. Finally, we want to mention another approach to the problem. In [42], Torres, motivated by the study of the propagation of electromagnetic waves through a multilayered optical medium, proved the existence of two different kinds of homoclinic solutions to the origin in a Schrödinger equation with a nonlinear term, by using a fixed-point theorem in cones.

Our purpose is to complete the mentioned bibliography with a variant of the cubicquintic nonlinear Schrödinger equation. The cubic-quintic nonlinear Schrödinger equation with inhomogeneous nonlinearities is

$$
-u^{\prime \prime}+\lambda u=a(x) u^{3}+b(x) u^{5}
$$


This equation can be seen as a particular case of the so-called nonpolynomial Schrödinger equation (NPSE) [43]. Really, in the case of weak nonlinearity, the NPSE can be expanded, which leads to a simplified $1 D$ equation with a combination of cubic and quintic terms [44]. In this form, we can obtain (1.5).

Equation (1.5) has a lot of applications to the mean field theory for Bose-Einstein condensates [45] and nonlinear optics [4]. We want to remark that in the free space, that is, $a(x)=4$ and $b(x)=3 \sigma$, where $\sigma= \pm 1$ (see, e.g., [46]), (1.5) has a localized exact solution:

$$
u(x)=\frac{\lambda^{1 / 2}}{(1+\sqrt{1+\sigma \lambda} \cosh (2 \sqrt{\lambda} x))^{1 / 2}} .
$$

On the other hand, it is known that for (1.5) some soliton solutions become bistable (i.e., singular solitons with the same carried power but different propagation parameters) [47-50].

An interesting problem arises when we consider the time-dependent cubic-quintic nonlinear Schrödinger equation

$$
i \psi_{t}=-\psi_{x x}-g_{1}(x)|\psi|^{2} \psi-g_{2}(x)|\psi|^{4} \psi
$$

since the problem of collapse appears. In the presence of the self-focusing quintic term, collapse is inevitable, and it may affect the stability of solitons against small perturbations.

It is known that (1.7), for $g_{i}(x) \equiv C_{i}=$ constant, $i=1,2$ (homogeneous case), has no blowup solution in the class

$$
\left\{\psi \in H^{1}(\mathbb{R}) \mid\|\psi(0, x)\|_{L^{2}}<\|R\|_{L^{2}}\right\},
$$

where $R$ is the ground state of the equation

$$
-u^{\prime \prime}+\lambda u-C_{1} u^{3}-C_{2} u^{5}=0 .
$$

In the class

$$
\left\{\psi \in H^{1}(\mathbb{R}) \mid\|\psi(0, x)\|_{L^{2}}=\|R\|_{L^{2}}\right\},
$$

one has that (1.7) has a unique blowup solution (see, e.g., [14]).

In any case, in this paper, we focus on the proof of the existence of solutions of (1.5), and we will not consider the problem of collapse and stability of the solutions of (1.7), which is an open problem in the case of inhomogeneous nonlinearity and will be studied elsewhere.

Thus, in this paper, we will prove the existence of bright solitons for (1.5). To do it, we will use critical point theory (the mountain pass theorem). Thus, we will use a variational approach to our equation, and prove that it satisfies the conditions of the mountain pass theorem. Moreover, using bifurcation theory, we will prove that when the chemical potential $\lambda \rightarrow 0^{+},\left\|u_{\lambda}\right\|^{2} \rightarrow 0$. Finally, we will solve this equation by using a numerical scheme, called imaginary time method.

The rest of the paper is organized as follows. In Section 2, we use a variational approach to the stationary cubic-quintic nonlinear Schrödinger equation with inhomogeneous nonlinearity, and some preliminary results are collected. Section 3 contains the main result about the existence of positive solutions. Section 4 deals with a result about bifurcation theory. Finally, Section 5 contains a numerical scheme to solve the time-dependent cubic-quintic nonlinear Schrödinger equation with inhomogeneous nonlinearity. 


\section{The variational approach}

In this paper, we will study the cubic-quintic nonlinear Schrödinger equation with inhomogeneous nonlinearities $g_{i}(x), i=1,2$ (CQINLSE), on $\mathbb{R}$, that is,

$$
i \psi_{t}=-\psi_{x x}-g_{1}(x)|\psi|^{2} \psi-g_{2}(x)|\psi|^{4} \psi,
$$

with $g_{i}: \mathbb{R} \rightarrow \mathbb{R}, i=1,2$, which satisfies the following properties:

$$
g_{i} \in L^{\infty}(\mathbb{R}), \quad g_{i}(x)>0, \quad \lim _{|x| \rightarrow \infty} g_{i}(x)=0, \quad i=1,2 .
$$

The solitary wave solutions of (2.1) are given by $\psi(x, t)=e^{i \lambda t} u(x)$, where $u(x)$ is the solution of

$$
-u_{x x}+\lambda u=g_{1}(x) u^{3}+g_{2}(x) u^{5},
$$

which can be identified as bright solitons due to their boundary conditions:

$$
u(x) \longrightarrow 0 \quad \text { as } x \longrightarrow \pm \infty .
$$

In this paper, we search for positive solutions to $\lambda>0$. Thus, the following theorem gives the existence of positive solitary waves, for $\lambda>0$.

Theorem 2.1 (existence of a positive solution). When $\lambda>0$, (2.3) has a positive solution $u \in$ $H^{1}(\mathbb{R})$.

In order to prove this theorem, we will introduce a set of preparatory definitions and lemmas. Formally, (2.3) is the Euler-Lagrange equation of the functional $J: H^{1}(\mathbb{R}) \rightarrow \mathbb{R}$, defined by

$$
J(u)=\frac{1}{2} \int_{\mathbb{R}}\left[\left|u_{x}\right|^{2}+\lambda|u|^{2}\right] d x-\frac{1}{4} \int_{\mathbb{R}} g_{1}(x)|u|^{4} d x-\frac{1}{6} \int_{\mathbb{R}} g_{2}(x)|u|^{6} d x .
$$

We define

$$
\begin{aligned}
\|u\|^{2} & =\int_{\mathbb{R}}\left|u_{x}\right|^{2}+\lambda|u|^{2} d x, \\
\Psi_{1}(u) & =\frac{1}{4} \int_{\mathbb{R}} g_{1}(x)|u|^{4} d x, \\
\Psi_{2}(u) & =\frac{1}{6} \int_{\mathbb{R}} g_{2}(x)|u|^{6} d x .
\end{aligned}
$$

We can therefore rewrite the functional (2.5) in the following way:

$$
J(u)=\frac{1}{2}\|u\|^{2}-\Psi_{1}(u)-\Psi_{2}(u) .
$$

Remark that $H^{1}(\mathbb{R}) \hookrightarrow L^{p}(\mathbb{R}), p \geq 2$, and thus $J$ is well defined on $H^{1}(\mathbb{R})$ and is smooth. It is very easy to check that, for each fixed $\lambda>0,\|\cdot\|$ is an equivalent norm to that which is usual in $H^{1}(\mathbb{R})$. Clearly, $J$ is of $C^{2}$ class and its critical points give rise to solutions of (2.3) such that $\lim _{|x| \rightarrow \infty} u=0$. 


\section{Existence of a positive solution}

In order to obtain critical points of $J$, we will use the mountain pass theorem [51]. This theorem deals with the existence of critical points of a functional $J \in\left(C^{1}(E), \mathbb{R}\right)$, where $E$ is a Hilbert space (although, in general, $E$ can be a Banach space), which satisfies the following two "geometric" assumptions.

$\left(\mathrm{MP}_{1}\right)$ There exist $r, \rho>0$ such that $J(u) \geq \rho$, for all $u \in E$, with $\|u\|=r$.

$\left(\mathrm{MP}_{2}\right)$ There exists $v \in E,\|v\|>r$, such that $J(v) \leq 0=J(0)$.

Moreover, it assumes the compactness condition $(\mathrm{PS})_{c}$, called the Palais-Smale condition at level $c$.

Every sequence $u_{n}$ such that

(1) $J\left(u_{n}\right) \rightarrow c$,

(2) $J^{\prime}\left(u_{n}\right) \rightarrow 0$

has a converging subsequence. $J^{\prime}(u)$ is called the derivative of $J$ at $u$, which exists by the Riesz theorem and is given by the following expression:

$$
\left(J^{\prime}(u) \mid \zeta\right)=(u \mid \zeta)-\left(\Psi_{1}^{\prime}(u) \mid \zeta\right)-\left(\Psi_{2}^{\prime}(u) \mid \zeta\right), \quad \forall \zeta \in H^{1}(\mathbb{R}),
$$

where

$$
\begin{aligned}
& \left(\Psi_{1}^{\prime}(u) \mid \zeta\right)=\int_{\mathbb{R}} g_{1}(x) u^{3} \zeta, \\
& \left(\Psi_{2}^{\prime}(u) \mid \zeta\right)=\int_{\mathbb{R}} g_{2}(x) u^{5} \zeta .
\end{aligned}
$$

The sequences satisfying (1),(2) are called (PS) $c$ sequences.

Consider the class of all the paths joining $u=0$ and $u=v$ :

$$
\Gamma=\{\gamma \in C([0,1], E): \gamma(0)=0, \gamma(1)=v\},
$$

and set

$$
c=\inf _{\gamma \in \Gamma} \max _{t \in[0,1]} J(\gamma(t))
$$

with $t$ being the variable of the curve $\gamma$. For reader's convenience, we will enunciate a simplified version of the mountain pass theorem (see [51] for the general setting).

Theorem 3.1 (mountain pass theorem). If $J \in C^{1}(E, \mathbb{R})$ satisfies the geometric conditions (1.1) and $(1.2)$ and the $(P S)_{c}$ Palais-Smale condition holds, then $c$ is a positive critical level for J. Precisely, there exists $z \in E$ such that $J(z)=c>0$ and $J^{\prime}(z)=0$. In particular, $z \neq 0$ and $z \neq v$.

Consider the following lemma.

Lemma 3.2. The functional J satisfies the geometric assumptions of the mountain pass theorem. 
Proof. (1) From the definition of $J$, the hypothesis (2.2) on $g_{i}, i=1,2$, and the Sobolev embedding $H^{1}(\mathbb{R}) \hookrightarrow L^{p}(\mathbb{R}), p \geq 2$ (see, e.g., [36]), we obtain

$$
J(u)=\frac{1}{2}\|u\|^{2}-\Psi_{1}(u)-\Psi_{2}(u) \geq \frac{1}{2}\|u\|^{2}-C\|u\|^{4}-M\|u\|^{6},
$$

where $C$ and $M$ are positive constants. As a consequence, there exist $r, \rho>0$ such that

$$
J(u) \geq \rho, \quad \forall u \in H^{1}(\mathbb{R}), \text { with }\|u\|=r,
$$

which proves that $J$ verifies $\left(\mathrm{MP}_{1}\right)$.

(2) Consider $v_{0} \in H^{1}(\mathbb{R}) \backslash\{0\}$, and let $s$ be a parameter such that for $s>0$,

$$
J_{\lambda}\left(s v_{0}\right)=\frac{s^{2}}{2}\left\|v_{0}\right\|_{\lambda}^{2}-s^{4} \Psi_{1}\left(v_{0}\right)-s^{6} \Psi_{2}\left(v_{0}\right) \searrow-\infty \text { as } s \nearrow \infty
$$

As a consequence, taking $v=s_{0} v_{0}$ with $s_{0} \gg 1$, we obtain $J_{\lambda}(v)<0=J_{\lambda}(0)$. It follows that $J(s v) \rightarrow-\infty$ as $s \rightarrow+\infty$.

In order to apply the mountain pass theorem (2), we have to study $(\mathrm{PS})_{c}$ sequences.

Lemma 3.3. Palais-Smale sequences are bounded.

Proof. From condition (1) of the definition of (PS) sequences, $J\left(u_{n}\right) \leq k$ and we obtain

$$
\left\|u_{n}\right\|^{2} \leq 2 k+2 \Psi_{1}\left(u_{n}\right)+2 \Psi_{2}\left(u_{n}\right) .
$$

From $J^{\prime}\left(u_{n}\right) \rightarrow 0$ and using the definition of $J^{\prime}$, we infer

$$
\left|\left\|u_{n}\right\|^{2}-4 \Psi_{1}\left(u_{n}\right)-6 \Psi_{2}\left(u_{n}\right)\right|=\left|\left(J^{\prime}\left(u_{n}\right) \mid u_{n}\right)\right| \leq\left\|J^{\prime}\left(u_{n}\right)\right\|\left\|u_{n}\right\|=\epsilon\left\|u_{n}\right\|,
$$

for $\epsilon>0$. Thus,

$$
\int_{\mathbb{R}} g_{1}(x) u_{n}^{4}+\int_{\mathbb{R}} g_{2}(x) u_{n}^{6} \leq\left\|u_{n}\right\|^{2}+\epsilon\left\|u_{n}\right\| .
$$

Using (3.8), we obtain

$$
\begin{aligned}
\left\|u_{n}\right\|^{2} & \leq 2 k+\frac{1}{2} \int_{\mathbb{R}} g_{1}(x) u_{n}^{4}+\frac{1}{3} \int_{\mathbb{R}} g_{2}(x) u_{n}^{6} \\
& \leq 2 k+\frac{1}{2}\left[\int_{\mathbb{R}} g_{1}(x) u_{n}^{4}+\int_{\mathbb{R}} g_{2}(x) u_{n}^{6}\right] \\
& \leq 2 k+\frac{1}{2}\left\|u_{n}\right\|^{2}+\frac{\epsilon}{2}\left\|u_{n}\right\|,
\end{aligned}
$$

and thus, for all $n$ and some constant $k$, we deduce that

$$
\frac{1}{2}\left\|u_{n}\right\|^{2} \leq 2 k+\frac{\epsilon}{2}\left\|u_{n}\right\|
$$

and the boundedness of (PS) sequences follows. 
Lemma 3.4. $\Psi_{i}$ is weakly continuous and $\Psi_{i}^{\prime}$ is compact, for each $i=1,2$.

Proof. Let $u_{n} \rightarrow u$ weakly in $H^{1}(\mathbb{R})$. As any weakly convergent sequence is bounded, that is, there exists a constant $K>0$ such that $\left\|u_{n}\right\|_{H^{1}(\mathbb{R})} \leq K$, then, according to the Sobolev imbedding theorem, constants $C, M>0$ must exist such that $\left\|u_{n}\right\|_{L^{4}(\mathbb{R})}<C$ and $\left\|u_{n}\right\|_{L^{6}(\mathbb{R})}<M$. So, given $\epsilon>0$, from condition (2.2), it follows that there exist $R_{1}, R_{2}>0$ such that

$$
\begin{aligned}
& \int_{|x| \geq R_{1}} g_{1}(x)\left(\left|u_{n}\right|^{4}-|u|^{4}\right) d x \leq \epsilon, \\
& \int_{|x| \geq R_{2}} g_{2}(x)\left(\left|u_{n}\right|^{6}-|u|^{6}\right) d x \leq \epsilon .
\end{aligned}
$$

On the other hand, let $B_{R_{1}}$ and $B_{R_{2}}$ be the open balls of radii $R_{1}$ and $R_{2}$, respectively. Since $H^{1}\left(B_{R_{1}}\right)$ is compactly embedded in $L^{4}\left(B_{R_{1}}\right)$ and $H^{1}\left(B_{R_{2}}\right)$ is also compactly embedded in $L^{6}\left(B_{R_{2}}\right)$, we have that $u_{n} \rightarrow u$ strongly in $L^{4}\left(B_{R_{1}}\right)$ and $L^{6}\left(B_{R_{2}}\right)$, respectively. Moreover, there exists a constant $M>0$ such that

$$
\begin{aligned}
& \left|\left[\int_{|x| \leq R_{1}} g_{1}(x)\left|u_{n}\right|^{4} d x\right]^{1 / 4}-\left[\int_{|x| \leq R_{1}} g_{1}(x)|u|^{4} d x\right]^{1 / 4}\right| \\
& \quad=\left|\left\|g_{1}^{1 / 4} u_{n}\right\|_{L^{4}\left(B_{R_{1}}\right)}-\left\|g_{1}^{1 / 4} u\right\|_{L^{4}\left(B_{R_{1}}\right)}\right| \leq\left|\left\|g_{1}^{1 / 4}\left(u_{n}-u\right)\right\|_{L^{4}\left(B_{R_{1}}\right)}\right| \leq M\left\|u_{n}-u\right\|_{L^{4}\left(B_{R_{1}}\right)} \leq \epsilon
\end{aligned}
$$

for $\epsilon>0$ and a sufficiently large $n$. It is easy to check that a similar inequality exists for $g_{2}(x)$. Putting together the two preceding inequalities, it follows that $\Psi_{i}, i=1,2$, is weakly continuous.

The proof that $\Psi_{i}^{\prime}, i=1,2$, is compact is similar. Let

$$
\left\|\Psi_{1}^{\prime}\left(u_{n}\right)-\Psi_{1}^{\prime}(u)\right\|=\sup _{\|\varphi\| \leq 1}\left\{\int g_{1}(x)\left(\left|u_{n}\right|^{3}-|u|^{3}\right) \varphi d x\right\}
$$

for $\varphi \in H^{1}(\mathbb{R})$. Using the Holder inequality, we obtain

$$
\left\|\Psi_{1}^{\prime}\left(u_{n}\right)-\Psi_{1}^{\prime}(u)\right\| \leq\left\|g_{1}(x)\left(\left|u_{n}\right|^{3}-|u|^{3}\right)\right\|_{L^{p}(\mathbb{R})}\|\varphi\|_{L^{q}(\mathbb{R})},
$$

with $1 / p+1 / q=1, p \geq 2, q<\infty$. Using the arguments previously exposed, we immediately show that

$$
\left\|g_{1}(x)\left(\left|u_{n}\right|^{3}-|u|^{3}\right)\right\|_{L^{p}(\mathbb{R})} \leq \epsilon,
$$

for $n \gg 1$ and $\epsilon>0$. This shows that $\Psi_{1^{\prime}}$ is a compact operator. The proof for $\Psi_{2}$ is similar.

We are now prepared to prove Theorem 2.1.

Let $u_{n}$ be a sequence that verifies the Palais-Smale conditions (1) and (2). Since $\left\|u_{n}\right\| \leq K$, we have that $u_{n} \rightarrow u$ weakly in $H^{1}(\mathbb{R})$. According to Lemma 3.4, $\Psi_{i}^{\prime}, i=1,2$, is compact. Therefore, there exists a subsequence, still denoted as $u_{n}$, such that $\Psi_{i}^{\prime}\left(u_{n}\right) \rightarrow \Psi_{i}^{\prime}(u)$. On the other hand, we know that

$$
J^{\prime}(u)=u-\Psi_{1}^{\prime}(u)-\Psi_{2}^{\prime}(u)
$$


Hence, we deduce that

$$
u_{n}=J^{\prime}\left(u_{n}\right)+\Psi_{1}^{\prime}\left(u_{n}\right)+\Psi_{2}^{\prime}\left(u_{n}\right)
$$

As $J^{\prime}\left(u_{n}\right) \rightarrow 0$, since $u_{n}$ is a Palais-Smale sequence, we obtain

$$
u_{n} \longrightarrow \Psi_{1}^{\prime}(u)+\Psi_{2}^{\prime}(u)
$$

proving that (PS) ${ }_{c}$ holds for every $c$.

We can thus apply the mountain pass theorem (2) since the conditions of this theorem are satisfied, and there exists $u_{\curlywedge} \in H^{1}(\mathbb{R})$ such that $J\left(u_{\curlywedge}\right)=c$ and $J^{\prime}\left(u_{\curlywedge}\right)=0$. The positivity is clear, using the maximum principle.

\section{Bifurcation from the positive solution}

By using the bifurcation theory (see, e.g., $[52,53]$ for an introduction to bifurcation theory), we can investigate the behavior of the positive solution $u_{\curlywedge}$ when $\lambda \searrow 0$.

Let $c(\lambda)$ denote the mountain pass critical level of $J$ for the positive solution $u_{\lambda}$. We suppose that $g_{i}(x), i=1,2$, moreover to verify condition (2.2), satisfies the following: there exist $C_{i}>0, K_{i}>0, i=1,2$, and $\tau_{1}>1$ and $\tau_{2} \in(0,1)$ such that

$$
g_{i}(x) \geq K_{i}|x|^{-\tau_{i}}, \quad \forall|x| \geq C_{i}, i=1,2 .
$$

Thus, we are able to prove the following lemma.

Lemma 4.1. If $g_{i}(x), i=1,2$, satisfies conditions (2.2) and (4.1), then $c(\lambda) \rightarrow 0$ as $\lambda \rightarrow 0^{+}$.

Proof. Fix the function $\Phi(x)=|x| e^{-|x|}$ and set $u_{\alpha}(x)=\Phi(\alpha x)$. There hold

$$
\begin{aligned}
& \left\|u_{\alpha}^{\prime}\right\|_{L^{2}(\mathbb{R})}^{2}=\alpha A_{1}, \quad A_{1}=\int_{\mathbb{R}}\left|\Phi^{\prime}\right|^{2} d x, \\
& \left\|u_{\alpha}\right\|_{L^{2}(\mathbb{R})}^{2}=\alpha^{-1} A_{2}, \quad A_{2}=\int_{\mathbb{R}} \Phi^{2} d x .
\end{aligned}
$$

One thus finds that

$$
\left\|u_{\alpha}\right\|^{2}=\frac{1}{2}\left\|u_{\alpha}^{\prime}\right\|_{L^{2}(\mathbb{R})}^{2}+\lambda\left\|u_{\alpha}\right\|_{L^{2}(\mathbb{R})}^{2}=\frac{1}{2} A_{1} \alpha+\lambda A_{2} \alpha^{-1} .
$$

If we take $\lambda=\alpha^{2}$, then we obtain $\left\|u_{\alpha}\right\|^{2}=A_{3} \alpha, \lambda>0$, for $A_{3}=1 / 2 A_{1}+A_{2}>0$. Moreover, by using (4.1), we deduce

$$
\begin{aligned}
& \Psi_{1}\left(u_{\alpha}\right)=\frac{1}{4} \int_{\mathbb{R}} g_{1}(x)\left|u_{\alpha}(x)\right|^{4} d x \geq \frac{1}{4} K_{1} \int_{|x| \geq C_{1}}|x|^{-\tau_{1}}\left|u_{\alpha}(x)\right|^{4} d x, \\
& \Psi_{2}\left(u_{\alpha}\right)=\frac{1}{6} \int_{\mathbb{R}} g_{2}(x)\left|u_{\alpha}(x)\right|^{6} d x \geq \frac{1}{6} K_{2} \int_{|x| \geq C_{2}}|x|^{-\tau_{2}}\left|u_{\alpha}(x)\right|^{6} d x .
\end{aligned}
$$


By changing variable $y=\alpha x$, we discover that

$$
\begin{aligned}
& \int_{|x| \geq C_{1}}|x|^{-\tau_{1}}|\Phi(\alpha x)|^{4} d x=\int_{|y| \geq C_{1} \alpha}\left|\frac{y}{\alpha}\right|^{-\tau_{1}}|\Phi(y)|^{4} \alpha^{-1} d y \geq \alpha^{\tau_{1}-1} \int_{|y| \geq C_{1}}|y|^{-\tau_{1}}|\Phi(y)|^{4} d x, \\
& \int_{|x| \geq C_{2}}|x|^{-\tau_{2}}|\Phi(\alpha x)|^{6} d x=\int_{|y| \geq C_{2} \alpha}\left|\frac{y}{\alpha}\right|^{-\tau_{2}}|\Phi(y)|^{6} \alpha^{-1} d y \geq \alpha^{\tau_{2}-1} \int_{|y| \geq C_{2}}|y|^{-\tau_{2}}|\Phi(y)|^{6} d x,
\end{aligned}
$$

where the domain of integration can be changed since $0<\alpha \leq 1$. Therefore, there exist $A_{5}>0$ and $A_{6}>0$ such that $\Psi_{1}\left(u_{\alpha}\right) \geq K_{1} \alpha^{\tau_{1}-1} A_{5} / 4$ and $\Psi_{2}\left(u_{\alpha}\right) \geq K_{2} \alpha^{\tau_{2}-1} A_{6} / 6$. Putting together all the preceding estimates, we obtain

$$
J\left(u_{\alpha}\right)=\frac{1}{2}\left\|u_{\alpha}\right\|^{2}-\Psi_{1}\left(u_{\alpha}\right)-\Psi_{2}\left(u_{\alpha}\right) \leq \frac{1}{2} A_{3} \alpha-\frac{K_{1} A_{5}}{4} \alpha^{\tau_{1}-1}-\frac{K_{2} A_{6}}{6} \alpha^{\tau_{2}-1} .
$$

Recall that

$$
c(\lambda)=\inf _{\gamma \in \Gamma} \max _{0 \leq t \leq 1} J(\gamma(t))
$$

where $\Gamma$ is the class of all paths joining 0 and $v, J(v) \leq 0$. Let us consider the following continuous curve:

$$
\begin{aligned}
r:[0,1] & \longrightarrow H^{1}(\mathbb{R}) \\
t & \longrightarrow t M u_{\alpha}
\end{aligned}
$$

where $M$ is a constant, $M \gg 1$. It is clear that $\gamma$ is a path in $\Gamma$ since it joins 0 and $v_{\alpha}=M u_{\alpha}$ and $J\left(M u_{\alpha}\right) \leq 0$, for sufficiently large $M$. Then,

$$
c(\lambda) \leq \max _{0 \leq t \leq 1} J\left(t M u_{\alpha}\right) \quad\left(\lambda=\alpha^{2}\right) .
$$

We now use the above estimate to evaluate $\max _{0 \leq t \leq 1} J\left(t M u_{\alpha}\right)$. There holds

$$
J\left(t M u_{\alpha}\right) \leq \beta(t)=\frac{1}{2} A_{3} t^{2} M^{2} \alpha-\frac{1}{4} K_{1} A_{5} \alpha^{\tau_{1}-1} t^{4} M^{4}-\frac{1}{6} K_{2} A_{6} \alpha^{\tau_{2}-1} t^{6} M^{6} .
$$

The maximum of $\beta$ is achieved at

$$
t_{\max }= \pm M \sqrt{\frac{-K_{1} A_{5} \alpha^{\tau_{1}-\tau_{2}}}{2 K_{2} A_{6}}+\sqrt{\frac{K_{1}^{2} A_{5}^{2} \alpha^{2 \tau_{1}-2 \tau_{2}}}{4 K_{2}^{2} A_{6}^{2}}+\frac{\alpha^{1-\tau_{2}}}{K_{2} A_{6}}}}
$$

Therefore,

$$
c(\lambda) \leq \frac{1}{2} A_{3} t_{\max }^{2} M^{2} \alpha-\frac{1}{4} K_{1} A_{5} \alpha^{\tau_{1}-1} t_{\max }^{4} M^{4}-\frac{1}{6} K_{2} A_{6} \alpha^{\tau_{2}-1} t_{\max }^{6} M^{6},
$$

with $\lambda=\alpha^{2}$. Since $\tau_{1}>1$ and $\tau_{2} \in[0,1)$, we find that $c(\lambda) \rightarrow 0$ as $\lambda=\alpha^{2} \rightarrow 0^{+}$. 
We have proved that the MP critical point $u_{\lambda}$ satisfies $J\left(u_{\lambda}\right)=c(\lambda) \rightarrow 0$ as $\lambda \rightarrow 0^{+}$.

Moreover, multiplying (2.3) by $u_{\lambda}$, using the fact that $u^{\prime}( \pm \infty)=0$, since $u( \pm \infty)=0$ and by integration, we obtain

$$
\int_{\mathbb{R}}\left|u_{\lambda}\right|^{2} d x+\lambda \int_{\mathbb{R}} u_{\lambda}^{2} d x=\int_{\mathbb{R}} g_{1}(x) u_{\lambda}^{4} d x+\int_{\mathbb{R}} g_{2}(x) u_{\lambda}^{6} d x
$$

or

$$
J\left(u_{\curlywedge}\right)=\Psi_{1}\left(u_{\curlywedge}\right)+2 \Psi_{2}\left(u_{\curlywedge}\right) .
$$

As $J\left(u_{\lambda}\right) \rightarrow 0$ as $\lambda \rightarrow 0^{+}$, it must be verified that $\Psi_{1}\left(u_{\curlywedge}\right) \rightarrow 0$ and $\Psi_{2}\left(u_{\curlywedge}\right) \rightarrow 0$ as $\lambda \rightarrow 0^{+}$. Thus, $\left\|u_{\lambda}\right\| \rightarrow 0$ as $\lambda \rightarrow 0^{+}$.

We have proved that when $\lambda \rightarrow 0^{+},\left\|u_{\lambda}\right\|^{2} \rightarrow 0$. In fact, we can prove that, for $\lambda=0$, if there exist solutions which are different from the trivial ones, these solutions would have an infinite amount of nodes. To do so, we will first prove the nonexistence of positive solutions of the equation

$$
-u_{x x}-g_{1}(x) u^{3}-g_{2}(x) u^{5}=0, \quad u( \pm \infty)=0 .
$$

Let $u$ be a strict positive solution of (4.15). Then,

$$
u_{x x}=-g_{1}(x) u^{3}(x)-g_{2}(x) u^{5}<0, \quad \forall x \in \mathbb{R} .
$$

Let $x_{0}$ be a global maximum point of the solution $u$, that is, $u\left(x_{0}\right)=\max _{x \in \mathbb{R}} u(x)>0$. This maximum point clearly exists owing to the boundary conditions. Then, $u^{\prime}\left(x_{0}\right)=0$ and, moreover, $u^{\prime \prime}(x)<0$, for all $x \in \mathbb{R}$. Then, from $\left[x_{0},+\infty\right), u$ must be decreasing and therefore $u^{\prime}(x)<0$, for all $x \in\left[x_{0},+\infty\right)$. Then, $u$ must cross the $x$-axis since $u^{\prime \prime}(x)<0$, for all $x \in \mathbb{R}$, which contradicts the initial hypothesis.

As

$$
\begin{array}{ll}
u_{x x}=-g_{1}(x) u^{3}(x)-g_{2}(x) u^{5}(x)<0 & \text { if } u>0, \\
u_{x x}=-g_{1}(x) u^{3}(x)-g_{2}(x) u^{5}(x)>0 & \text { if } u<0,
\end{array}
$$

it is clear that the solution has an infinite amount of nodes.

\section{Numerical method}

In this section, we will solve (2.1) by using an imaginary time method. This method allows us to calculate the positive solution, or ground state, of (2.3).

First, we have the change $t \rightarrow-i t$ in (2.1). Thus, we obtain

$$
u_{t}=u_{x x}+g_{1}(x)|u|^{2}+g_{2}(x)|u|^{4} u
$$

We must study (5.1) numerically. To this end, we have developed a Fourier pseudospectral scheme for the discretization of the spatial derivatives combined with a split-step scheme to compute the time evolution. Split-step schemes are based on the observation that many problems may be decomposed into exactly solvable parts and on the fact that the full problem 
may be approximated as a composition of the individual problems. For instance, the solution of partial differential equations of the type $\partial_{t} u(x, t)=N\left(t, x, u, \partial_{x}, \ldots\right) u=(A+B) u$ can be approximated from the exact solutions of the problems $\partial_{t} u=A u$ and $\partial_{t} u=B u$.

Let us decompose the evolution operator in (5.1) by taking $[54,55]$

$$
\begin{gathered}
A=\partial_{x x}, \\
B=g_{1}(x)|u|^{2}+g_{2}(x)|u|^{4} .
\end{gathered}
$$

To proceed with split-step-type methods, it is necessary to compute the explicit form of the operators $e^{\left(t-t_{0}\right) A}$ and $e^{\left(t-t_{0}\right) B}$. To obtain the action of the operators, we solve the subproblems

$$
\begin{gathered}
\partial_{t} u=\partial_{x x} u, \\
\partial_{t} u=g_{1}(x)|u|^{2} u+g_{2}(x)|u|^{4} u .
\end{gathered}
$$

Thus, after some algebra and defining the time step $\tau$ as $\tau=\left(t-t_{0}\right) / c, c \in R$, after a suitable renaming, we obtain the explicit form of the operators

$$
\begin{gathered}
e^{\left(t-t_{0}\right) A} \equiv e^{c \tau A}=\mathcal{F}^{-1} e^{-k^{2} c \tau} \mathcal{F}, \\
e^{\left(t-t_{0}\right) B} \equiv e^{c \tau B}=e^{\left(g_{1}(x)|u|^{2}+g_{2}(x)|u|^{4}\right)\left(t-t_{0}\right)},
\end{gathered}
$$

where $\mathcal{F}$ denotes the spatial Fourier transform. As we now have the explicit form of the solutions of subproblems (5.3), we are able to obtain the positive solution of (2.1) to any degree of accuracy. We have used the second-order splitting classical method whose equation is

$$
u(x, t+\tau)=e^{\tau A / 2} e^{\tau B} e^{\tau A / 2} u(x, t)+\mathcal{O}\left(\tau^{3}\right) .
$$

This scheme has many advantages. First, it is more accurate than the numerical methods based on finite difference [56]. Second, from the practical point of view, the calculation of the Fourier transform, which is the most computer time-consuming step in the calculations, may be done by using the fast Fourier transform (FFT). Thus, the computational cost of the method is of order $O\left(N^{2} \log N\right)$, with $N$ being the points' number in each spatial direction of the grid which is quite acceptable. The use of discrete transforms to represent the continuous Fourier transform in (5.5) implicitly imposes periodic boundary conditions on $u$. However, since $u$ is expected to be negligible on the boundaries (otherwise the computational domain must be enlarged), this is not an essential point. Another convenient property of this scheme is its preservation of the $L^{2}$-norm of the solutions.

Thus, the pictures in Figure 1 show the positive solutions of (2.1) for various $g_{1}(x), g_{2}(x)$ functions, which satisfy condition (2.2). For the first picture (Figure 1(a)),

$$
\begin{aligned}
& g_{1}(x)=\frac{C_{1}}{1+x^{2}}, \\
& g_{2}(x)=\frac{C_{2}}{1+x^{4}},
\end{aligned}
$$

with $C_{1}=-1$ and $C_{2}=-1$. For the second picture (Figure $1(\mathrm{~b})$ ),

$$
\begin{aligned}
& g_{1}(x)=C_{1} e^{-x^{2}}, \\
& g_{2}(x)=\frac{C_{2}}{1+2 x^{2}},
\end{aligned}
$$

with $C_{1}=-1$ and $C_{2}=-1$. 


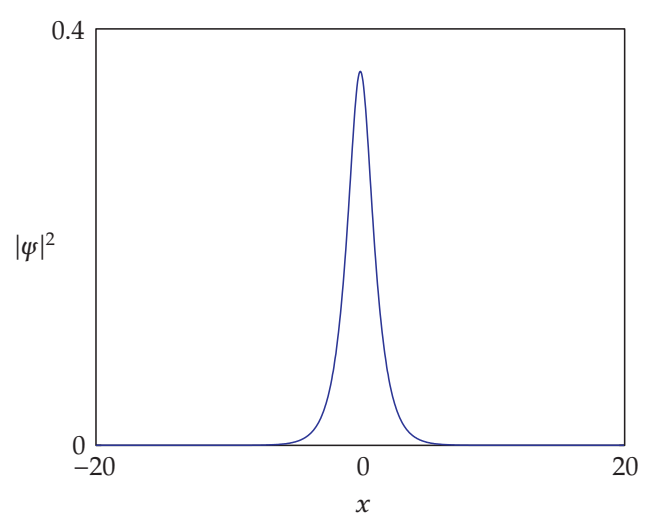

(a)

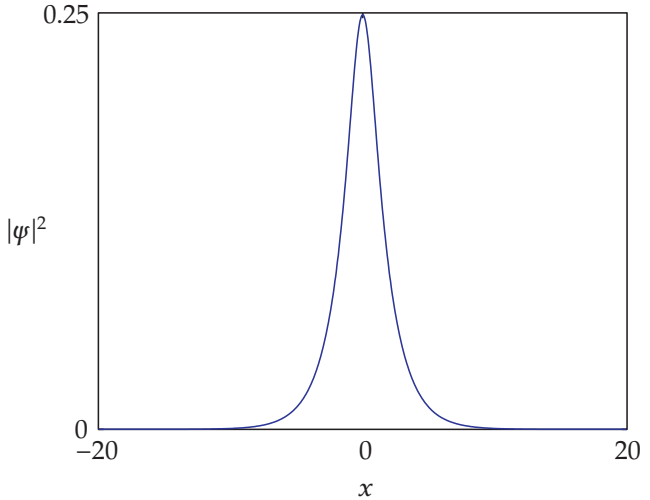

(b)

Figure 1: Some stationary solutions of (2.1) for (a) $g_{1}(x), g_{2}(x)$ functions given by (5.6), and (b) $g_{1}(x), g_{2}(x)$ functions given by (5.7) (see text).

\section{Conclusions}

In this paper, we have proved the existence of bright soliton or positive solutions of the cubicquintic nonlinear Schrödinger equations with inhomogeneous nonlinearities. In order to do this, we have used a variational approach and a critical point theory. Moreover, by using bifurcation theory, we have proved that the norm of the positive solution goes to zero as the parameter $\lambda$ tends to zero. Finally, by using an imaginary time method, we have numerically solved the inhomogeneous nonlinear Schrödinger equation for different nonlinearities.

\section{Acknowledgments}

The author would like to thank Professor E. Colorado for his helpful comments and for a first critical reading of the manuscript. He is also indebted to two anonymous referees for pointing out some inaccuracies in the first version of the paper and for providing some interesting references. This work has been supported by Grants nos. FIS2006-04190 (Ministerio de Educación y Ciencia, Spain) and PCI08-093 (Junta de Comunidades de Castilla-La Mancha, Spain).

\section{References}

[1] L. Vázquez, L. Streit, and V. M. Pérez-García, Eds., Nonlinear Klein-Gordon and Schrödinger Systems: Theory and Applications, World Scientific, Singapore, 1996.

[2] F. Brezzi and P. A. Markowich, "The three-dimensional Wigner-Poisson problem: existence, uniqueness and approximation," Mathematical Methods in the Applied Sciences, vol. 14, no. 1, pp. 35$61,1991$.

[3] J. L. Lopez and J. Soler, "Asymptotic behavior to the 3-D Schrödinger/Hartree-Poisson and WignerPoisson systems," Mathematical Models E Methods in Applied Sciences, vol. 10, no. 6, pp. 923-943, 2000.

[4] Y. S. Kivshar and G. P. Agrawal, Optical Solitons: From Fibers to Photonic Crystals, Academic Press, San Diego, Calif, USA, 2003.

[5] A. Hasegawa, Optical Solitons in Fibers, Springer, Berlin, Germany, 1989.

[6] R. K. Dodd, J. C. Eilbeck, J. D. Gibbon, and H. C. Morris, Solitons and Nonlinear Wave Equations, Academic Press, London, UK, 1982. 
[7] J. L. Rosales and J. L. Sánchez-Gómez, "Nonlinear Schödinger equation coming from the action of the particle's gravitational field on the quantum potential," Physics Letters A, vol. 166, no. 2, pp. 111-115, 1992.

[8] R. Fedele, G. Miele, L. Palumbo, and V. G. Vaccaro, "Thermal wave model for nonlinear longitudinal dynamics in particle accelerators," Physics Letters A, vol. 179, no. 6, pp. 407-413, 1993.

[9] F. Dalfovo, S. Giorgini, L. P. Pitaevskii, and S. Stringari, "Theory of Bose-Einstein condensation in trapped gases," Reviews of Modern Physics, vol. 71, no. 3, pp. 463-512, 1999.

[10] B. A. Malomed, Soliton Management in Periodic Systems, Springer, New York, NY, USA, 2006.

[11] A. S. Davydov, Solitons in Molecular Systems, vol. 4 of Mathematics and Its Applications, D. Reidel, Dordrecht, The Netherlands, 1985.

[12] A. Scott, Nonlinear Science: Emergence and Dynamics of Coherent Structures, vol. 1 of Oxford Texts in Applied and Engineering Mathematics, Oxford University Press, Oxford, UK, 1999.

[13] V. E. Zaharov, V. S. L'vov, and S. S. Starobinets, "Spin-wave turbulence beyond the parametric excitation threshold," Soviet Physics Uspekhi, vol. 17, no. 6, pp. 896-919, 1975.

[14] C. Sulem and P. Sulem, The Nonlinear Schrödinger Equation: Self-Focusing and Wave Collapse, Springer, Berlin, Germany, 2000.

[15] G. Fibich and G. Papanicolau, "Self-focusing in the perturbed and unperturbed nonlinear Schrödinger equation in critical dimension," SIAM Journal on Applied Mathematics, vol. 60, no. 1, pp. 183-240, 1999.

[16] M. I. Rodas-Verde, H. Michinel, and V. M. Pérez-García, "Controllable soliton emission from a BoseEinstein condensate," Physical Review Letters, vol. 95, no. 15, Article ID 153903, 4 pages, 2005.

[17] A. Vázquez-Carpentier, H. Michinel, M. I. Rodas-Verde, and V. M. Pérez-García, "Analysis of an atom soliton laser based on the spatial control of the scattering length," Physical Review A, vol. 74, no. 1, Article ID 013619, 8 pages, 2006.

[18] F. K. Abdullaev and J. Garnier, "Propagation of matter-wave solitons in periodic and random nonlinear potentials," Physical Review A, vol. 72, no. 6, Article ID 061605, 4 pages, 2005.

[19] G. Theocharis, P. Schmelcher, P. G. Kevrekidis, and D. J. Frantzeskakis, "Matter-wave solitons of collisionally inhomogeneous condensates," Physical Review A, vol. 72, no. 3, Article ID 033614, 9 pages, 2005.

[20] J. Garnier and F. K. Abdullaev, "Transmission of matter-wave solitons through nonlinear traps and barriers," Physical Review A, vol. 74, no. 1, Article ID 013604, 6 pages, 2006.

[21] M. T. Primatarowa, K. T. Stoychev, and R. S. Kamburova, "Interaction of solitons with extended nonlinear defects," Physical Review E, vol. 72, no. 3, Article ID 036608, 5 pages, 2005.

[22] M. A. Porter, P. G. Kevrekidis, B. A. Malomed, and D. J. Frantzeskakis, "Modulated amplitude waves in collisionally inhomogeneous Bose-Einstein condensates," Physica D, vol. 229, no. 2, pp. 104-115, 2007.

[23] H. Sakaguchi and B. A. Malomed, "Matter-wave solitons in nonlinear optical lattices," Physical Review E, vol. 72, no. 4, Article ID 046610, 8 pages, 2005.

[24] Y. Sivan, G. Fibich, and M. I. Weinstein, "Waves in nonlinear lattices: ultrashort optical pulses and Bose-Einstein condensates," Physical Review Letters, vol. 97, no. 19, Article ID 193902, 4 pages, 2006.

[25] G. Theocharis, P. Schmelcher, P. G. Kevrekidis, and D. J. Frantzeskakis, "Dynamical trapping and transmission of matter-wave solitons in a collisionally inhomogeneous environment," Physical Review A, vol. 74, no. 5, Article ID 053614, 5 pages, 2006.

[26] F. Abdullaev, A. Abdumalikov, and R. Galimzyanov, "Gap solitons in Bose-Einstein condensates in linear and nonlinear optical lattices," Physics Letters A, vol. 367, no. 1-2, pp. 149-155, 2007.

[27] A. Ambrosetti, V. Felli, and A. Malchiodi, "Ground states of nonlinear Schrödinger equations with potentials vanishing at infinity," Journal of the European Mathematical Society, vol. 7, no. 1, pp. 117-144, 2005.

[28] A. Ambrosetti and E. Colorado, "Standing waves of some coupled nonlinear Schrödinger equations," Journal of the London Mathematical Society, vol. 75, no. 1, pp. 67-82, 2007.

[29] A. Ambrosetti, E. Colorado, and D. Ruiz, "Multi-bump solitons to linearly coupled systems of nonlinear Schrödinger equations," Calculus of Variations and Partial Differential Equations, vol. 30, no. 1, pp. 85-112, 2007.

[30] A. Bahri and P.-L. Lions, "On the existence of a positive solution of semilinear elliptic equations in unbounded domains," Annales de l'Institut Henri Poincaré. Analyse Non Linéaire, vol. 14, no. 3, pp. 365413, 1997. 
[31] T. Bartsch and Z. Q. Wang, "Existence and multiplicity results for some superlinear elliptic problems on $\mathbb{R}^{N}$," Communications in Partial Differential Equations, vol. 20, no. 9-10, pp. 1725-1741, 1995.

[32] H. Berestycki and P.-L. Lions, "Nonlinear scalar field equations-I: existence of a ground state," Archive for Rational Mechanics and Analysis, vol. 82, no. 4, pp. 313-345, 1983.

[33] H. Berestycki and P.-L. Lions, "Nonlinear scalar field equations-II: existence of infinitely many solutions," Archive for Rational Mechanics and Analysis, vol. 82, no. 4, pp. 347-375, 1983.

[34] P.-L. Lions, "The concentration-compactness principle in the calculus of variations. The locally compact case-I," Annales de l'Institut Henri Poincaré. Analyse Non Linéaire, vol. 1, no. 2, pp. 109-145, 1984.

[35] P.-L. Lions, "The concentration-compactness principle in the calculus of variations. The limit caseII," Annales de l'Institut Henri Poincaré. Analyse Non Linéaire, vol. 1, no. 2, pp. 223-283, 1984.

[36] M. Willem, Minimax Theorems, vol. 24 of Progress in Nonlinear Differential Equations and Their Applications, Birkhäuser, Boston, Mass, USA, 1996.

[37] T. Bartsch and M. Willem, "Infinitely many radial solutions of a semilinear elliptic problem on $\mathbb{R}^{N}$," Archive for Rational Mechanics and Analysis, vol. 124, no. 3, pp. 261-276, 1993.

[38] T. Bartsch and A. Szulkin, "Hamiltonian systems: periodic and homoclinic solutions by variational methods," in Handbook of Differential Equations: Ordinary Differential Equations. Vol II, pp. 77-146, Elsevier, Amsterdam, The Netherlands, 2005.

[39] P. Korman and A. C. Lazer, "Homoclinic orbits for a class of symmetric Hamiltonian systems," Electronic Journal of Differential Equations, no. 1, pp. 1-10, 1994.

[40] J. Belmonte-Beitia, V. M. Pérez-García, V. Vekslerchik, and P. J. Torres, "Lie symmetries and solitons in nonlinear systems with spatially inhomogeneous nonlinearities," Physical Review Letters, vol. 98, no. 6, Article ID 064102, 4 pages, 2007.

[41] J. Belmonte-Beitia, V. M. Pérez-García, V. Vekslerchik, and P. J. Torres, "Lie symmetries, qualitative analysis and exact solutions of nonlinear Schrödinger equations with inhomogeneous nonlinearities," Discrete and Continuous Dynamical Systems. Series B, vol. 9, no. 2, pp. 221-233, 2008.

[42] P. J. Torres, "Guided waves in a multi-layered optical structure," Nonlinearity, vol. 19, no. 9, pp. 21032113, 2006.

[43] L. Salasnich, A. Parola, and L. Reatto, "Effective wave equations for the dynamics of cigar-shaped and disk-shaped Bose condensates," Physical Review A, vol. 65, no. 4, Article ID 043614, 6 pages, 2002.

[44] L. Salasnich, A. Cetoli, B. A. Malomed, F. Toigo, and L. Reatto, "Bose-Einstein condensates under a spatially modulated transverse confinement," Physical Review A, vol. 76, no. 1, Article ID 013623, 10 pages, 2007.

[45] L. Pitaevskii and S. Stringari, Bose-Einstein Condensation, vol. 116 of International Series of Monographs on Physics, The Clarendon Press, Oxford University Press, Oxford, UK, 2003.

[46] D. E. Pelinovsky, Y. S. Kivshar, and V. V. Afanasjev, "Internal modes of envelope solitons," Physica D, vol. 116, no. 1-2, pp. 121-142, 1998.

[47] A. E. Kaplan, “Bistable solitons,” Physical Review Letters, vol. 55, no. 12, pp. 1291-1294, 1985.

[48] S. Gatz and J. Herrmann, "Soliton propagation in materials with saturable nonlinearity," Journal of the Optical Society of America B, vol. 8, no. 11, pp. 2296-2302, 1991.

[49] J. Herrmann, "Bistable bright solitons in dispersive media with a linear and quadratic intensitydependent refraction index change," Optics Communications, vol. 87, no. 4, pp. 161-165, 1992.

[50] H. Michinel, "Secondary bistable solitons in non-linear gradient index media," Optical and Quantum Electronics, vol. 28, no. 8, pp. 1013-1019, 1996.

[51] A. Ambrosetti and P. H. Rabinowitz, "Dual variational methods in critical point theory and applications," Journal of Functional Analysis, vol. 14, no. 4, pp. 349-381, 1973.

[52] A. Ambrosetti and A. Malchiodi, Perturbation Methods and Semilinear Elliptic Problems on $R^{n}$, vol. 240 of Progress in Mathematics, Birkhäuser, Basel, Switzerland, 2006.

[53] A. Ambrosetti and A. Malchiodi, Nonlinear Analysis and Semilinear Elliptic Problems, vol. 104 of Cambridge Studies in Advanced Mathematics, Cambridge University Press, Cambridge, UK, 2007.

[54] V. M. Pérez-García and X. Liu, "Numerical methods for the simulation of trapped nonlinear Schrödinger systems," Applied Mathematics and Computation, vol. 144, no. 2-3, pp. 215-235, 2003.

[55] G. D. Montesinos and V. M. Pérez-García, "Numerical studies of stabilized Townes solitons," Mathematics and Computers in Simulation, vol. 69, no. 5-6, pp. 447-456, 2005.

[56] L. N. Trefethen, Spectral Methods in MATLAB, vol. 10 of Software, Environments, and Tools, SIAM, Philadelphia, Pa, USA, 2000. 


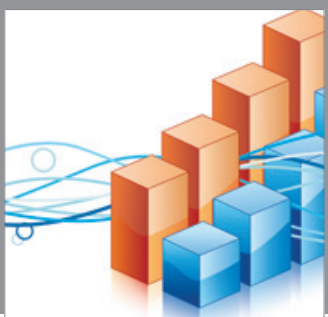

Advances in

Operations Research

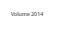

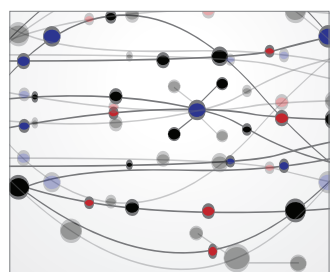

\section{The Scientific} World Journal
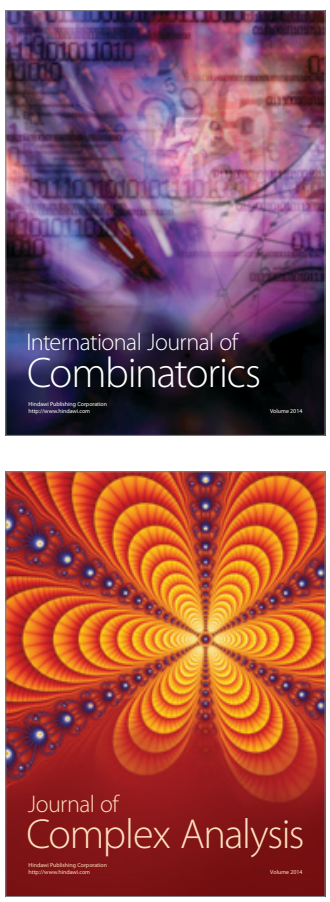

International Journal of

Mathematics and

Mathematical

Sciences
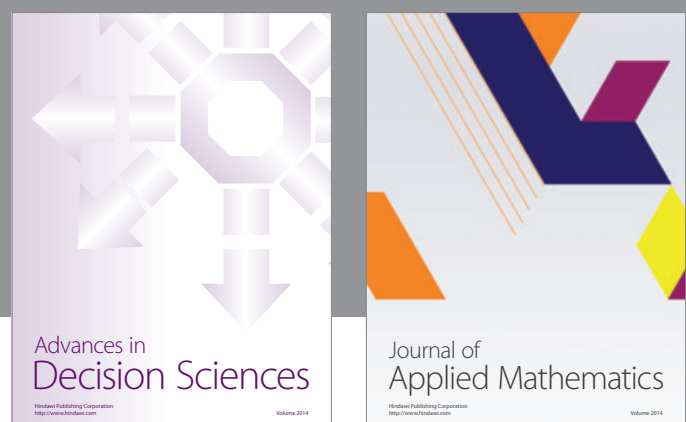

Journal of

Applied Mathematics
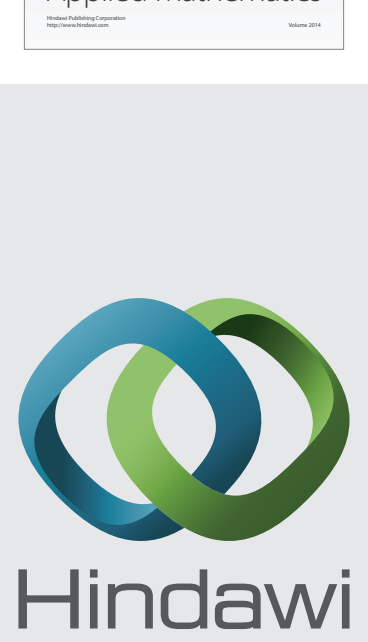

Submit your manuscripts at http://www.hindawi.com
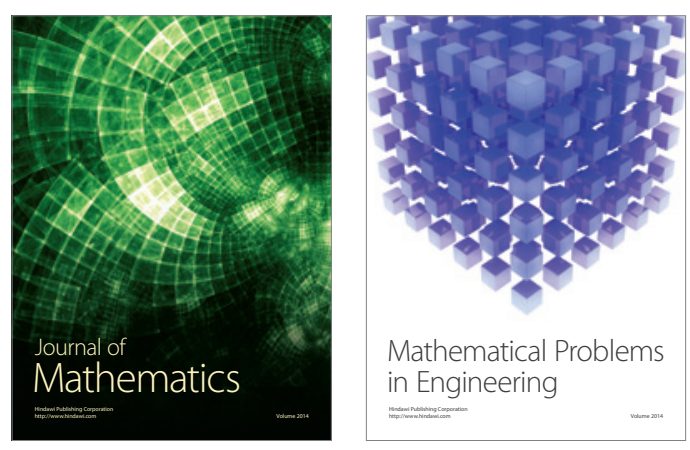

Mathematical Problems in Engineering
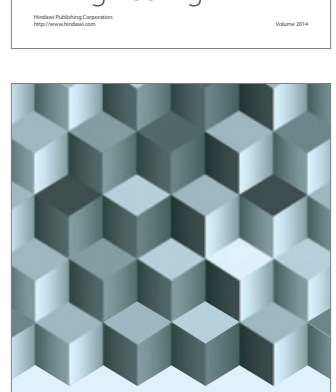

Journal of

Function Spaces
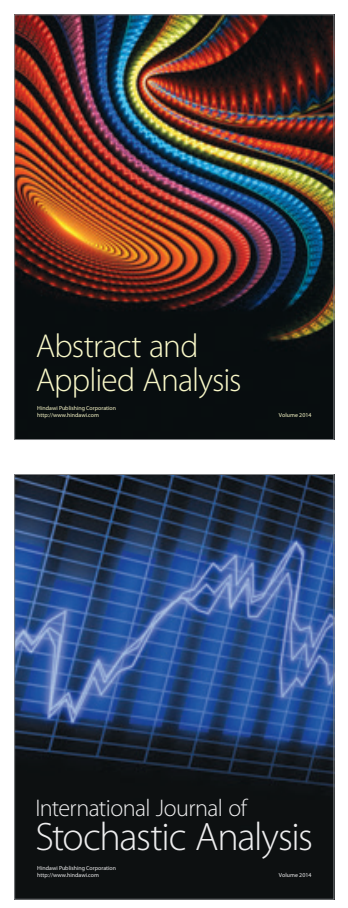

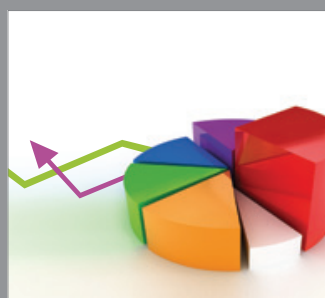

ournal of

Probability and Statistics

Promensencen
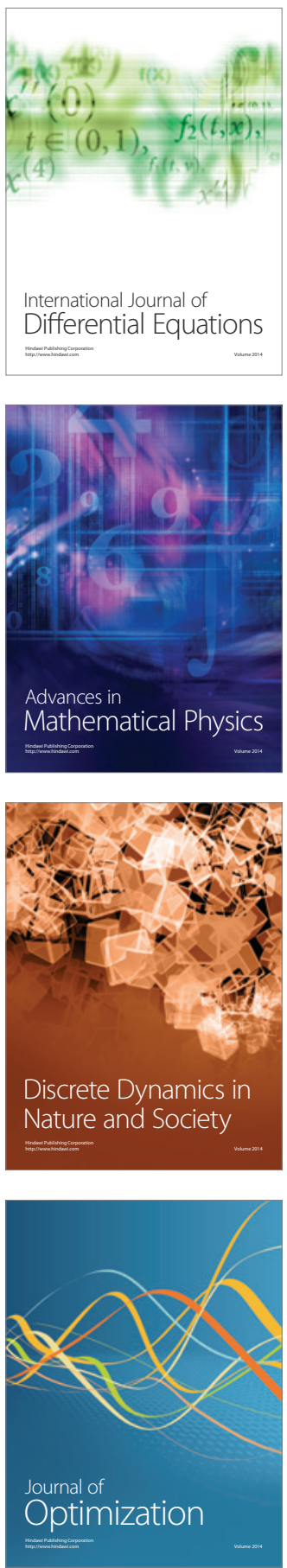